July 24, 2021

OHSTPY-HEP-T-94-023

LBL-36357,

UCB-PTH-94/27

\title{
A Complete Supersymmetric SO(10) Model
}

\author{
Lawrence J. Hall ^ \\ Department of Physics, University of California at Berkeley \\ and \\ Theoretical Physics Group, Lawrence Berkeley Laboratory \\ 1 Cyclotron Road, Berkeley, California 94720 \\ hall_lj@theorm.lbl.gov \\ and \\ Stuart Raby i] \\ Department of Physics, The Ohio State University \\ 174 W. 18th Ave., Columbus, OH 43210 \\ raby@mps.ohio-state.edu
}

\begin{abstract}
A complete supersymmetric $\mathrm{SO}(10)$ model is constructed, which is the most general consistent with $R$, discrete, and $U(1)$ flavor symmetries. At the supersymmetric level there are many degenerate vacua, one of which is phenomenologically successful. This vacuum has vevs which align in certain definite directions in $\mathrm{SO}(10)$ group space, such as the $B-L$ direction. Although this desired vacuum is not proven to be the global minimum of the entire theory, including supersymmetry breaking, it is separated from vacua where the vevs point in
\end{abstract}

*Supported in part by U.S. Department of Energy contract DE- AC03-76SF00098, and by the National Science Foundation under grant PHY-90-21139

${ }^{\dagger}$ Supported in part by U.S. Department of Energy contract DE-ER-01545-640. 
different group directions by a large potential barrier. This desired vacuum simultaneously leads to three major features of the theory. (1) $\mathrm{SO}(10)$ is broken at scale $v_{10}$ to $\mathrm{SU}(5)$, which breaks at $M_{G}$ to the standard model gauge group. Beneath $M_{G}$ the only light gauge non-singlet fields are those of the minimal supersymmetric standard model, so that the successful prediction for the weak mixing angle is retained. (2) The alignment of the vevs leads to a natural mass separation of the weak Higgs doublets from their colored partners via a mechanism which is closely related to the issue of the proton decay rate. Also, the generation of the $\mu$ term is studied. (3) A set of particles acquire mass at the highest perturbative scale of the theory, $M$ and at $v_{10}$. When they are integrated out, they lead to just four flavor operators for quark and charged lepton masses, and two more for neutrino masses. These flavor operators lead to many quark and lepton mass and mixing angle relations which involve pure $\mathrm{SO}(10)$ group theory numerical Clebsch factors. The family hierarchies result from the ratio of scales $v_{10} / M, M_{G} / v_{10}$ and $M_{G} / M$. While the theory is by no means unique, it is complete, and hence illustrates the close connecton between several important features of the theory. 


\section{Disclaimer}

This document was prepared as an account of work sponsored by the United States Government. While this document is believed to contain correct information, neither the United States Government nor any agency thereof, nor The Regents of the University of California, nor any of their employees, makes any warranty, express or implied, or assumes any legal liability or responsibility for the accuracy, completeness, or usefulness of any information, apparatus, product, or process disclosed, or represents that its use would not infringe privately owned rights. Reference herein to any specific commercial products process, or service by its trade name, trademark, manufacturer, or otherwise, does not necessarily constitute or imply its endorsement, recommendation, or favoring by the United States Government or any agency thereof, or The Regents of the University of California. The views and opinions of authors expressed herein do not necessarily state or reflect those of the United States Government or any agency thereof or The Regents of the University of California and shall not be used for advertising or product endorsement purposes.

Lawrence Berkeley Laboratory is an equal opportunity employer. 


\section{Introduction}

The Standard Model is a great success. In the coming years experiments will continue to look for deviations from the Standard Model which might indicate new physics. There are already several suggestive hints of new physics.

- The measured values of $\alpha, \sin ^{2} \theta_{W}$ and $\alpha_{s}\left(M_{Z}\right)$ are consistent with the prediction of a simple supersymmetric[SUSY] grand unified the$\operatorname{ory}[\mathrm{GUT}]$ (with $\alpha_{i} \approx \alpha_{G}, \mathrm{i}=1,2,3$, at $M_{G} \sim 10^{16} \mathrm{GeV}$ and a soft SUSY breaking scale of order $\left.(1-10) \times M_{Z}\right)$ [四].

- The measured values of $m_{b}, m_{\tau}$ and $m_{t}$ are consistent with SUSY GUTs (with the Yukawa couplings satisfying the $\mathrm{SU}(5)$ relation $\lambda_{b}=\lambda_{\tau}$ at $\left.M_{G}\right)$. This constraint correlates $m_{t}$ and the ratio of Higgs vacuum expectation values[vevs], $\tan \beta$. For $m_{t} \sim 174 \mathrm{GeV}$, there is both a small and large solution for $\tan \beta$. In fact, the $\mathrm{SO}(10)$ relation $\lambda_{b}=\lambda_{\tau}=\lambda_{t}$ at $M_{G}$ is also consistent with observation [2]. This constraint favors the solution with $\tan \beta$ in the range $40-60$.

- The cosmological evidence for a universe filled with cold and hot dark matter fits nicely into the minimal supersymmetric standard model[MSSM] with a conserved R-parity. In this case the lightest SUSY particle is absolutely stable and is an excellent candidate for the cold dark matter constituent. A tau neutrino with mass of order several $\mathrm{eV}$ is the natural candidate for the hot dark matter. Such a tau neutrino may be observable at the CHORUS and NOMAD experiments at CERN and at the E803 experiment at Fermilab.

- Cosmological and astrophysical evidence for neutrino masses and mixing angles is indicative of right-handed neutrino components which naturally fit into an $\mathrm{SO}(10)$ framework for fermions.

\footnotetext{
${ }^{\ddagger}$ Recently it has been shown that acceptable values for $m_{b} / m_{\tau}$ together with a right-
} 
All of these hints are quite tantalizing. As a whole they strongly suggest the notion of a SUSY GUT. It is an intriguing question then whether the symmetry relations of a SUSY GUT can help us understand the observed pattern of fermion masses and mixing angles. Recently, several effective $\mathrm{SO}(10)$ SUSY GUT models were found which provide a consistent and quantitative description of this low energy data [4] (from now on referred to as paper I) [5].

$\mathrm{SO}(10)$ [6] has the advantage that it is the minimal GUT in which all the fermions in one family, i.e. $\mathrm{u}, \mathrm{d}$, e and $\nu$, fit into one irreducible representation with only one additional state - a right-handed neutrino. Thus mass matrices in different charge sectors can be related. In reference I a search was made for all acceptable $\mathrm{SO}(10)$ flavor sectors in which all quark and charged lepton masses and mixings originated in just four operators. Several such models of flavor were found to be consistent with data. This agreement may of course be completely spurious, or these operators may give the dominant contributions to fermion masses in the effective theory at $M_{G}$. If the latter is true then corrections to the leading order results should improve this agreement. Why should only a few operators contribute to fermion masses? Why should nature be kind and allow us to make many flavor predictions without addressing physics at the Planck scale? This is an important criticism of reference I. The answer given in this paper is that flavor symmetries of the theory just beneath the Planck scale can be very powerful, forbidding the vast majority of possible operaters.

The main purpose of this paper is to provide a framework in which such corrections may be calculated. There are several sources of corrections: electroweak scale threshold corrections must be included, which can be significant for large $\tan \beta$. GUT scale threshold corrections and GUT scale corrections to the effective theory can affect fermion masses as well as FCNC processes.

handed neutrino Majorana mass of order $10^{12} \mathrm{GeV}$ (as indicated, for example, by a tau neutrino component of dark matter) requires large $\tan \beta$ [3]. 
In addition, new phenomena such as proton decay and neutrino masses can only be addressed in the context of a complete SO(10) SUSY GUT valid for energies greater than $M_{G}$ up to the largest perturbative scale in the theory, $M$, which can be taken as the string compactification scale or the Planck mass.

In this paper we demonstrate the existence of a complete $\mathrm{SO}(10) \mathrm{SUSY}$ GUT which reproduces, as an example, one of the models found in I. By a complete GUT we mean one which reproduces not only a realistic fermion mass spectrum (including neutrinos) but also addresses the issues of GUT symmetry breaking, the doublet-triplet splitting problem, the $\mu$ problem and proton decay. We show how all these problems have reasonable solutions, with one caveat. We do not claim to understand the dynamics which determines the scale of the GUT vevs. All GUT breaking occurs along flat directions in the SUSY limit. We will also not address the question of the origin of soft SUSY breaking. We will just assume the standard set of soft SUSY breaking terms below $M$ whenever they are needed to make contact with low energy data.

We make no claim of uniqueness for the model in this paper: there are undoubtedly many similar such models. Nevertheless, by studying a specific, complete model, one can study the connections between many different aspects of the theory. The orientation of the GUT vevs is crucial for understanding both the lightness of the Higgs doublets and for obtaining the operators which lead to predictions for the quark and lepton masses. Similarly, the doublet-triplet splitting problem is intimately connected with the proton decay rate. Finally, a family symmetry, which is imposed to give some understanding of the observed pattern of quark and lepton masses and mixings, has important consequences for many other aspects of the theory.

In the next section we discuss the flavor sector of the effective theory at $M_{G}$. It involves just four operators, and we study how this structure may emerge from symmetries of the full theory. In sections 3 and 4 we focus on the GUT symmetry breaking and Higgs potential respectively. Section 4 
also includes the mechanisms for doublet-triplet splitting, for solving the $\mu$ problem and constraints from proton decay. In section 5 we consider neutrino masses. Finally in section 6, we discuss the full set of symmetries of the theory. In section 7, we give our general conclusions and directions for further work.

\section{Charged Fermion Masses}

We take the flavor sector of our model to be that of model 6 in paper I. The fermion mass and mixing angle predictions which result from this flavor sector, for a particular choice of input parameters, are shown in Table I, reproduced from paper I. 
Table 1: Particular Predictions for Model 6 with $\alpha_{s}\left(M_{Z}\right)=0.115$

\begin{tabular}{|c|c|c|c|}
\hline $\begin{array}{c}\text { Input } \\
\text { Quantity }\end{array}$ & $\begin{array}{c}\text { Input } \\
\text { Value }\end{array}$ & $\begin{array}{c}\text { Predicted } \\
\text { Quantity }\end{array}$ & $\begin{array}{c}\text { Predicted } \\
\text { Value }\end{array}$ \\
\hline$m_{b}\left(m_{b}\right)$ & $4.35 \mathrm{GeV}$ & $M_{t}$ & $176 \mathrm{GeV}$ \\
$m_{\tau}\left(m_{\tau}\right)$ & $1.777 \mathrm{GeV}$ & $\tan \beta$ & 55 \\
\hline$m_{c}\left(m_{c}\right)$ & $1.22 \mathrm{GeV}$ & $V_{c b}$ & .048 \\
\hline$m_{\mu}$ & $105.6 \mathrm{MeV}$ & $V_{u b} / V_{c b}$ & .059 \\
$m_{e}$ & $0.511 \mathrm{MeV}$ & $m_{s}(1 G e V)$ & $172 \mathrm{MeV}$ \\
$V_{u s}$ & 0.221 & $\hat{B_{K}}$ & 0.64 \\
& & $m_{u} / m_{d}$ & 0.64 \\
& & $m_{s} / m_{d}$ & 24. \\
\hline
\end{tabular}

In addition to these predictions, the set of inputs in Table 1 predicts: $\sin 2 \alpha=$ $-.46, \sin 2 \beta=.49, \sin 2 \gamma=.84$, and $J=2.6 \times 10^{-5}$.

We have chosen model 6 of paper I since this model gives results which are in best agreement with the data. Model 9 of paper I generally gives values of $V_{c b}$ which are larger and model 4 seems to have too little CP violation. Of course these problems may in fact be solved by corrections to these leading order results.

The flavor sector is specified by a particular set of four operators $\left\{\mathrm{O}_{33}, \mathrm{O}_{32}\right.$, $\left.\mathrm{O}_{22}, \mathrm{O}_{12}\right\}$. Three of these operators $-\mathrm{O}_{33}, \mathrm{O}_{32}$, and $\mathrm{O}_{12}$ - are uniquely specified by choosing model 6 . On the other hand there are 6 choices for operator $\mathrm{O}_{22}$, as all give identical entries in the charged fermion Yukawa matrices. In order to construct the theory above $M_{G}$ one of these operators must be chosen. This ambiguity is real. Perhaps further study might reveal that only one of these operators is preferred by symmetry arguments. In the meantime we have made a particular selection.

The four effective fermion mass operators chosen are given by

$$
O_{33}=\quad 16_{3} 10_{1} 16_{3}
$$




$$
\begin{array}{lr}
O_{23}= & 16_{2} \frac{A_{2}}{\tilde{A}} 10_{1} \frac{A_{2}}{\tilde{A}} 16_{3} \\
O_{22}= & 16_{2} \frac{\tilde{A}}{\mathcal{S}_{M}} 10_{1} \frac{A_{1}}{\tilde{A}} 16_{2} \\
O_{12}= & 16_{1}\left(\frac{\tilde{A}}{\mathcal{S}_{M}}\right)^{3} 10_{1}\left(\frac{\tilde{A}}{\mathcal{S}_{M}}\right)^{3} 16_{2}
\end{array}
$$

The adjoint fields $A_{1}, A_{2}, \tilde{A}$ are assumed to get vevs equal to

$$
\begin{aligned}
\left\langle A_{1}\right\rangle & = & a_{1} \frac{3}{2}(B-L) \\
\left\langle A_{2}\right\rangle & = & a_{2} \frac{3}{2} Y \\
\langle\tilde{A}\rangle & = & -\tilde{a} X
\end{aligned}
$$

respectively with $a_{1}, a_{2} \sim M_{G}$ and $\tilde{a} \sim v_{10}>M_{G}$. The singlet $\mathcal{S}_{M}$ is assumed to get a vev $\sim M$. The superspace potential for these fields is discussed in the next section.

Consider the symmetries which are necessary in order to guarantee these and only these fermion mass operators. First it is clear that adjoints with distinct vevs appearing in the operators of eqn. 1 cannot be interchanged. Interchanging them would lead to different Clebschs and a new, unacceptable model. For each distinct vev we need one adjoint chiral supermultiplet. Also changing the position of the adjoints in each operator would alter the Clebschs and again lead to an unacceptable theory. In order to prevent interchangeability of the adjoints we assume they carry a different value of one (or possibly more) U(1) charges. However, we can only prevent the positional changes of adjoints in these operators by studying the symmetries of the theory above $v_{10}$, as we now demonstrate.

Each operator can be obtained via a unique tree diagram constructed with dimension 4 couplings to intermediate heavy 16 and $\overline{16}$ states. In fig. 1 we show the explicit decomposition for the effective operators of eqn. 1.

We now assign unique $\mathrm{U}(1)$ charges to the heavy $16, \overline{16}$ states such that no other dimension 4 couplings (other than those already appearing in the vertices of fig. 1) are consistent with the charge assignment. If this is possible then no other effective fermion mass operators can be obtained to leading 
order when integrating out the heavy $16, \overline{16}$ states. Note the heavy $16, \overline{16}$ states have mass greater than $M_{G}$ since the $\mathrm{SO}(10)$ singlet field $\mathcal{S}_{M}$ and adjoint $\tilde{A}$ are assumed to have vevs of order $M, v_{10}$ respectively with $M \geq$ $v_{10}>M_{G}$. The choice for $O_{22}$ will affect the possible $\mathrm{U}(1)$ charges of the intermediate states. Not all choices are allowed. Note also that the Higgs 10 coupling to the fermion mass operators carries the label 1. Two 10s are necessary for the doublet-triplet splitting mechanism used below, but only $10_{1}$ couples to fermions. This will be enforced by symmetries.

The superspace potential for this sector is given by

$$
\begin{aligned}
& W_{\text {fermion }}= \\
& 16_{3} 16_{3} 10_{1}+\bar{\Psi}_{1} A_{2} 16_{3}+\bar{\Psi}_{1} \tilde{A} \Psi_{1}+\Psi_{1} \Psi_{2} 10_{1} \\
& +\bar{\Psi}_{2} \tilde{A} \Psi_{2}+\bar{\Psi}_{2} A_{2} 16_{2}+\bar{\Psi}_{3} A_{1} 16_{2} \\
& +\bar{\Psi}_{3} \tilde{A} \Psi_{3}+\Psi_{3} \Psi_{4} 10_{1}+\mathcal{S}_{M} \sum_{a=4}^{9}\left(\bar{\Psi}_{a} \Psi_{a}\right) \\
& +\bar{\Psi}_{4} \tilde{A} 16_{2}+\bar{\Psi}_{5} \tilde{A} \Psi_{4}+\quad \bar{\Psi}_{6} \tilde{A} \Psi_{5} \\
& +\Psi_{6} \Psi_{7} 10_{1}+\bar{\Psi}_{7} \tilde{A} \Psi_{8}+\bar{\Psi}_{8} \tilde{A} \Psi_{9}+\bar{\Psi}_{9} \tilde{A} 16_{1}
\end{aligned}
$$

The form of this superpotential is guaranteed by the symmetries discussed in section 6 . In particular, an $R$ symmetry forces it to be trilinear.

\section{$3 \quad$ GUT Symmetry Breaking Sector}

The superspace potential for the adjoints $A_{1}, A_{2}, \tilde{A}$ must preserve their distinct $\mathrm{U}(1)$ charges. We also need 16 and $\overline{16}$ fields $\Psi, \bar{\Psi}$ to break the rank of $\mathrm{SO}(10)$ from 5 to 4 . Finally, all states of this sector which are non-singlets under the standard model gauge group must get mass $\geq M_{G}$. This is necessary so that the only states with mass $\leq M_{G}$ are in the MSSM or are singlets which don't affect the RG equations from $M_{G}$ to $M_{Z}$. These constraints are satisfied by the interactions

$$
W_{\text {symmetry breaking }}=
$$




$$
\begin{gathered}
A_{1}^{\prime}\left(S A_{1}+\mathcal{S}_{1} A_{1}\right)+S^{\prime}\left(\mathcal{S}_{2} S+A_{1}^{2}\right) \\
+\tilde{A}^{\prime}\left(\bar{\Psi} \Psi+\mathcal{S}_{3} \tilde{A}\right) \\
+A_{2}^{\prime}\left(\mathcal{S}_{4} A_{2}+S \tilde{A}+\left(\mathcal{S}_{1}+\mathcal{S}_{5}\right) \tilde{A}\right) \\
+\bar{\Psi}^{\prime} A_{2} \Psi+\bar{\Psi} A_{2} \Psi^{\prime} \\
+A_{1} A_{2} \tilde{A}^{\prime}+\mathcal{S}_{6}\left(A_{1}^{\prime}\right)^{2}
\end{gathered}
$$

where $S$ and $S^{\prime}$ are 54 dimensional representations; $\Psi, \Psi^{\prime}$ and $\bar{\Psi}, \bar{\Psi}^{\prime}$ are 16 and $\overline{16}$ respectively and $A_{1}, A_{2}, \tilde{A}, A_{1}^{\prime}, A_{2}^{\prime}, \tilde{A}^{\prime}$ are adjoints. The primed fields are necessary to preserve the distinct $\mathrm{U}(1)$ charge assignments for all fields. All primed fields are assumed to get zero vevs.

The superspace potential of eqn. 4 has many flat directions. Once SUSY is broken these flat directions will be lifted. In this paper we shall not speculate on how the combination of soft breaking terms, supergravity corrections and RG improved tree potential lifts this degeneracy to determine the GUT scale. We shall assume the necessary vevs and check that (a) they are consistent with a globally SUSY vacuum and (b) all states of this sector which are non-singlets under the standard model gauge group obtain mass of order $M_{G}$.

The SUSY vacua are specified by :

$$
\begin{gathered}
\left\langle A_{1}\right\rangle=a_{1}\left(\begin{array}{ccccc}
1 & & & & \\
& 1 & & & \\
& & 1 & & \\
& & & 0 & \\
& & & & 0
\end{array}\right) \otimes \eta \\
\left\langle A_{2}\right\rangle=a_{2}\left(\begin{array}{ccccc}
1 & & & & \\
& 1 & & & \\
& & 1 & & \\
& & & -3 / 2 & \\
& & & & -3 / 2
\end{array}\right) \otimes \eta
\end{gathered}
$$




$$
\begin{aligned}
& \langle\tilde{A}\rangle=\tilde{a}\left(\begin{array}{ccccc}
1 & & & & \\
& 1 & & & \\
& & 1 & & \\
& & & 1 & \\
& & & & 1
\end{array}\right) \otimes \eta \\
& \langle S\rangle=s\left(\begin{array}{ccccc}
1 & & & & \\
& 1 & & & \\
& & 1 & & \\
& & & -3 / 2 & \\
& & & & -3 / 2
\end{array}\right) \otimes \mathbf{1}
\end{aligned}
$$

where

$$
\eta=\left(\begin{array}{cc}
0 & -i \\
i & 0
\end{array}\right), \mathbf{1}=\left(\begin{array}{ll}
1 & 0 \\
0 & 1
\end{array}\right)
$$

In addition

$$
\langle\Psi\rangle=\langle\bar{\Psi}\rangle=V
$$

in the right-handed neutrino direction and

$$
\left\langle\mathcal{S}_{i}\right\rangle \neq 0
$$

for $i=1, \cdots, 7$. The vevs are constrained by the vacuum conditions

$$
\begin{gathered}
\mathcal{S}_{1}+s=0, \mathcal{S}_{2} s+\frac{2}{5} a_{1}^{2}=0, \frac{V^{2}}{4}+\mathcal{S}_{3} \tilde{a}=0 \\
\mathcal{S}_{4} a_{2}+s \tilde{a}=0, \mathcal{S}_{1}+\mathcal{S}_{5}=0 .
\end{gathered}
$$

The first term forces $A_{1}$ into either the $B-L$ or $T_{3 R}$ direction but has a GUT scale barrier to a linear combination of these two directions. Thus the $B-L$ vev is natural. This is made possible by the vev of $S$. The third term forces $\tilde{A}$ in the $X$ direction which is consistent with the $\mathrm{SU}(5)$ invariant vevs of $\Psi$ and $\bar{\Psi}$. The 4 th term allows $A_{2}$ to have a vev in some linear combination of $Y$ and $T_{3 R}$. Finally, the terms $\bar{\Psi} A_{2} \Psi^{\prime}, \bar{\Psi}^{\prime} A_{2} \Psi$ force $A_{2}$ into 
the $Y$ direction. All the other terms in eqn. 4 are necessary to give mass to all states of this sector which are non-singlets under the standard model gauge group.

Above the scale $v_{10}$ the gauge coupling is highly non-asymptotically free. The one loop evolved gauge coupling becomes non-perturbative at about $5 v_{10}$ (depending on both $v_{5} / v_{10}$ and on values of Yukawa couplings).

\section{Higgs Sector}

In the Higgs sector we distinguish between two cases which differ by the mechanism for solving the $\mu$ problem.

1. $\mu$ is generated by a D term coupling of the Higgs doublets to a hidden sector field $z$ whose F component breaks SUSY at an intermediate scale.

2. $\mu$ is generated by a one loop diagram containing soft SUSY breaking cubic scalar interactions. Thus $\mu$ is proportional to the soft SUSY breaking parameter $A$ [7].

In both cases, the reason for $\mu$ having a magnitude which is related to the scale of supersymmetry breaking is no longer a puzzle. The symmetries of the theory guarantee that the Higgs doublets are massless at tree level in the superpotential, and the $\mu$ term arises by introducing supersymmetry breaking into a higher dimension $\mathrm{D}$ operator. The two cases differ as to the origin of the D operator. In the first case it is present as a non-renormalizable operator from the Planck scale, while in the second case it is generated by a GUT-scale loop.

Higher dimension D operators generate the desired $\mu$ parameter so easily that its origin should not be considered a problem. Much more serious for grand unification is the large mass splitting required between the Higgs doublets and their color triplet partners. There are several mechanisms which have been invented to avoid the fine tunings of non-supersymmetric GUTs 
and of the Dimopoulos-Georgi supersymmetric GUT [1]. In this paper we use the Dimopoulos-Wilczek mechanism [8]. In the context of $\mathrm{SO}(10)$, this mechanism requires an adjoint field which points precisely in the $B-L$ direction. Our theory already contains such a field, $A_{1}$, with the vev shown in eqn. (2). This mechanism is a very natural choice in theories where vev alignments are needed to give fermion mass predictions.

\subsection{Case 1.}

In eqn. 5 we give the Lagrangian for the Higgs sector.

$$
L_{\text {Higgs }}=\left[10_{1} A_{1} 10_{2}+\mathcal{S}_{7} 10_{2}^{2}\right]_{F}+\frac{1}{M}\left[z^{*} 10_{1}^{2}\right]_{D}
$$

where $\mathcal{S}_{7}$ is assumed to get a non-zero vev. The first two terms (F terms) are necessary to incorporate the Dimopoulos-Wilczek mechanism for doublettriplet splitting. In the doublet sector the vev $A_{1}$ vanishes leaving two doublets massless. The massless Higgs doublets lie solely in the $10_{1}$. Thus the $\mathrm{SO}(10)$ relation $\lambda_{b} / \lambda_{t}=1$ remains exact. The last term is a $\mathrm{D}$ term and is necessary to solve the $\mu$ problem. The situation for proton decay is identical to case 2 below and so we save the discussion until then.

- $\mu$ term

In this case the hidden sector field $z$ is assumed to get a SUSY breaking vev such that $F_{z} \sim \mu M$.

\subsection{Case 2.}

In eqn. 6 we give the superspace potential for the Higgs sector.

$$
\begin{aligned}
& W_{\text {Higgs }}= 10_{1} A_{1} 10_{2}+\mathcal{S}_{7} 10_{2}^{2} \\
&+\bar{\Psi} \bar{\Psi}^{\prime} 10_{1}+\Psi \Psi^{\prime} 10_{1}
\end{aligned}
$$

The 3rd and 4th terms have been introduced solely to solve the $\mu$ problem.

- Doublet-Triplet Splitting 
The mass matrix for the $\mathrm{SU}(5) \overline{\mathbf{5}}, \mathbf{5}$ states in $\mathbf{1 0}_{\mathbf{1}}, \mathbf{1 0}_{\mathbf{2}}, \mathbf{\Psi}, \Psi^{\prime}, \overline{\mathbf{\Psi}}, \overline{\mathbf{\Psi}}^{\prime}$ is given below.

$$
\begin{aligned}
& \begin{array}{lllll}
\overline{5}_{1} & \overline{5}_{2} & \overline{5}_{\Psi} & \overline{5}_{\Psi^{\prime}}
\end{array} \\
& \begin{array}{c}
5_{1} \\
5_{2} \\
5_{\bar{\Psi}} \\
5_{\bar{\Psi}^{\prime}}
\end{array} \quad\left(\begin{array}{cccc}
0 & A_{1} & 0 & \Psi \\
A_{1} & \mathcal{S}_{7} & 0 & 0 \\
0 & 0 & 0 & A_{2} \\
\bar{\Psi} & 0 & A_{2} & 0
\end{array}\right)
\end{aligned}
$$

- Higgs doublets

In the doublet sector the vev $A_{1}$ vanishes leaving two doublets massless. The massless Higgs doublets, in this case, are a linear combination of the doublets in $10_{1}$ and those in $\Psi$ and $\bar{\Psi}$. As a result the boundary condition $\lambda_{b} / \lambda_{t}=1$ is corrected at tree level. The ratio is now given in terms of a ratio of mixing angles. In principle this could allow much smaller values of $\tan \beta$ than those predicted when $\lambda_{b} / \lambda_{t}=1$. However, in practice the predictions for $m_{u} / m_{d}$ and for the $\mathrm{CP}$ violating parameter $J$ require large $\tan \beta$ [9]. Electroweak symmetry breaking with large $\tan \beta$ can occur with a moderate fine tune of one part in $\tan \beta$ [10].

\section{- Proton decay}

The leading contribution to proton decay comes from the exchange of superheavy colored Higgsinos [11]. The rate for proton decay in this model is set by the quantity $\left(M^{t}\right)_{11}^{-1}$ where $M^{t}$ is the color triplet Higgsino mass matrix. We find $\left(M^{t}\right)_{11}^{-1}=\frac{\mathcal{S}_{7}}{A_{1}^{2}}$, which must be smaller than $\frac{1}{\tan \beta M_{G}}$ in order to be consistent with proton decay limits. Clearly this relation may be satisfied for $\mathcal{S}_{7}$ sufficiently smaller than $M_{G}$. Note there are no heavy color triplet states in this limit. Proton decay is suppressed because the color triplet Higgsinos in $10_{1}$ become Dirac fermions (with mass of order $M_{G}$ ), but they do not mix with each other. The ratio $\mathcal{S}_{7} / M_{G}$ cannot be taken too small however. This is because the doublets in $10_{2}$ are becoming lighter than their triplet partners. This will affect the RG running of the gauge couplings and is thus constrained by the low energy data. 
- $\mu$ term

In this case $\mu$ is generated at the one loop level, for example by the diagram of fig. 2. We find $\mu \sim \frac{\lambda^{4}}{16 \pi^{2}} A$. Thus $\mu$ is naturally smaller than the scale of SUSY breaking. This is a nice feature since it is consistent with a large $\tan \beta$ solution.

\section{$5 \quad$ Neutrino Masses}

Although right-handed neutrinos are included in the $16_{i}, i=1,2,3$ this does not mean that the three electroweak doublet (left-handed) neutrinos are massive.

An $R$ symmetry, discussed in the next section, forbids operators of the form $16_{i} \bar{\Psi} 16_{j} \bar{\Psi}$ which could generate Majorana masses for the right-handed neutrinos. To avoid Dirac neutrinos with weak-scale masses, extra fields must be introduced which couple to $\nu_{R}$ at the renormalizable level. The form of these interactions is very model dependent. In the simplest case the three left-handed neutrinos are massless. This is case 1 . In case 2 we show how to generate mass for the left-handed neutrinos.

\subsection{Case 1. - Massless Neutrinos}

In this case we introduce three $\mathrm{SO}(10)$ singlets fields $N_{i}, i=1,2,3$; one for each family. The superspace potential for neutrinos is given by

$$
W_{\text {neutrinos }}=\sum_{i=1}^{3}\left(16_{i} \bar{\Psi}\right) N_{i}
$$

When $\bar{\Psi}$ gets a vev the right-handed neutrinos mix with the singlet states to get Dirac masses of order $V$. The left-handed neutrinos remain massless. 


\subsection{Case 2. - Massive Neutrinos}

In this case there are several apriori acceptable possibilities. The different choices are described in terms of an effective symmetric mass matrix $M_{i j}=$ $M_{j i}$ for the states $N_{i}, i=1,2,3$. For every zero eigenvalue of $M_{i j}$ there is a corresponding massless neutrino. Thus with only one term, for example $M_{23}$, the $\mu$ and $\tau$ neutrinos would be massive and the electron neutrino would be massless. There would nevertheless be mixing among all three.

Choosing a set of operators will affect the symmetries of the theory. We can only accept operators which preserve enough symmetry to keep the theory natural. One example of an allowed neutrino mass sector is given by the following superspace potential. In this case we have effectively $M_{22}$ and $M_{13}$ non-zero.

$$
\begin{aligned}
W_{\text {neutrinos }}= & \sum_{i=1}^{3}\left(16_{i} \bar{\Psi}\right) N_{i} \\
+ & N_{2}^{2} \mathcal{S}_{M}+N_{1} N_{1}^{\prime} \mathcal{S}_{M} \\
+ & N_{3} N_{3}^{\prime} \mathcal{S}_{M}+N_{1}^{\prime} N_{3}^{\prime} \mathcal{S}
\end{aligned}
$$

where $\mathcal{S}=\mathcal{S}_{1}$ or $\mathcal{S}=\mathcal{S}_{3}$. Upon integrating out $\mathcal{S}_{M}$ we could obtain an equivalent description in terms of the higher dimension operators

$$
W_{\text {neutrinos }}=\frac{1}{\mathcal{S}_{M}}\left(16_{2} \bar{\Psi}\right)^{2}+\frac{1}{\mathcal{S}_{M}^{2}}\left(16_{1} \bar{\Psi}\right)\left(16_{3} \bar{\Psi}\right) \mathcal{S}
$$

Inserting $\bar{\Psi}$ vevs yields masses for the $\nu_{R}$ in the $16_{i}$, suppressed by various powers of $v_{10} / M$ and $M_{G} / M$. These masses take part in the see-saw mechanism giving small masses to the left-handed neutrinos. The suppression factors for the $\nu_{R}$ masses leads to enhancements of the masses of the light neutrinos, which can have phenomenologically important consequences. Since there are two operator coefficients in equation 9 , the 3 masses and 3 real mixing angles of the light neutrinos are given in terms of only 3 unknown parameters (two magnitudes and a phase). 


\section{Symmetries}

The theory of this paper is the most general consistent with several symmetries.

- Continuous R Symmetry — Since the theory is scale invariant it has a continuous $\mathrm{R}$ symmetry in which all superfields have charge 1 and the superspace potential has charge 3 . With this symmetry we need only consider dimension 4 operators in the superspace potential below $M$. This $R$ symmetry is a U(1) Peccei-Quinn symmetry. It solves the strong CP problem and leads to an invisible axion [12].

- Family Reflection Symmetry - This symmetry eliminates dimension 4 baryon number violating operators and is equivalent to $\mathrm{R}$ parity. Thus the LSP is stable. Under this symmetry all states in the set $\left\{16_{i}, N_{i}, i=\right.$ $\left.1,2,3 ; N_{1}^{\prime}, N_{3}^{\prime} ; \Psi_{a}, \bar{\Psi}_{a}, a=1, \cdots, 9\right\}$ are odd and all other states are even.

- Z(4) R Symmetry - This symmetry guarantees that only $10_{1}$ couples to fermions. Under this symmetry all primed fields, $\mathcal{S}_{6}, \mathcal{S}_{7}$ and $10_{2}$ are odd; the set $\left\{16_{i}, i=1,2,3 ; \Psi_{a}, \bar{\Psi}_{a}, a=1, \cdots, 9\right\}$ transform by $i \times\{\cdots\}$; all other fields are even.

- U(1) Flavor Symmetries. These symmetries guarantee that the dimension 4 operators included in equations $(3-8)$ are "natural" in the sense that no other dimension 4 operators are consistent with the family reflection symmetry, the Z(4) R symmetry and the U(1) symmetries. These U(1) symmetries are the only symmetries of the theory which distinguish between the three generations, $166_{i}$. They are largely responsible for determining which flavor operators are generated; hence, we call them flavor symmetries. The more terms in the superspace potential the fewer continuous symmetries exist. For example, consider the Higgs sector as in case 2 (eqn. 6) and the neutrino sector of eqn. (8). This theory has 49 fields and 47 superpotential interactions, hence there are two U(1) flavor symmetries. We have checked

\footnotetext{
§See Dimopoulos and Georgi [1].
} 
that these two symmetries rule out the addition of any new terms to W. The other Higgs sectors and neutrino sectors which we have discussed have fewer interactions, and correspondingly more $\mathrm{U}(1)$ symmetries.

The above symmetries are sufficient to limit the flavor interactions of the superpotential to those which lead to the desired fermion mass predictions. Furthermore, since all non-renormalizable $\mathrm{F}$ terms are forbidden, the predictions can be computed exactly in terms of the renormalizable coefficients. In general it is possible that higher dimension $\mathrm{D}$ operators, allowed by the above symmetries, could lead to corrections to the flavor predictions. However, if the supersymmetry breaking occurs in a hidden sector of a supergravity theory, flavor changing phenomenology leads to considerable constraints on the form of the higher dimension D operators. This phenomenology requires scalar masses and trilinear interactions to be close to universal, suggesting that the D terms have a trivial flavor structure, as would occur if they possessed a $U(N)$ invariance [13]. In this case the higher dimension $\mathrm{D}$ operators do not affect the fermion mass predictions. A further consequence of the $U(N)$ invariance of the Kähler potential is that the operator $\left[z^{*} 10_{1}^{2}\right]_{D}$ of equation (5), which was a possible source of the $\mu$ term, is absent. Hence, the $\mu$ term should be generated radiatively as in section (4.2).

In a supersymmetric theory, it may be questioned whether there is any need to guarantee the form of the desired interactions by symmetries. It has been argued that string theories can lead to low energy effective theories with interactions which are not the most general allowed by the low energy symmetries. This makes the task of model building easier. It is probable that a much simpler grand unifed $\mathrm{SO}(10)$ theory can be written down if

\footnotetext{
"We can envisage other schemes for the D terms. In the limit that the gaugino mass is much larger than the scalar mass and A terms at $M_{P}$, the flavor changing problem is alleviated and there is no need for universal scalar masses and A terms. In this case the $\mathrm{D}$ terms could give corrections to the fermion mass predictions, suppressed compared to the leading terms by powers of $v_{10} / M_{P}$. Also the $\mu$ term could occur via the operator $\left[z^{*} 10_{1}^{2}\right]_{D}$.
} 
one adopts this viewpoint. However, in a sense it makes model building too easy. For example, as a flavor sector one can simply write down the four flavor operators of equation 1. There is no symmetry to forbid all other operators, but no such symmetry is needed. Further operators can be added at will. While model building is much easier in this approach, it leads to much less understanding: the origin of flavor is hidden in physics above the string compactification scale. By contrast, in this paper flavor physics is determined by a set of global U(1) symmetries beneath the string scale 1

\section{Conclusions}

We have presented a complete $\mathrm{SO}(10)$ SUSY GUT incorporating the fermion mass predictions of model 6 of ref. [4]. We have addressed the issues of doublet-triplet splitting, proton decay, $\mu$ problem and fermion masses and mixing angles. In the SUSY limit the superspace potential has many flat directions, one of which appears consistent with low energy data. In particular, in this desired vacuum the GUT vevs align precisely in the directions necessary to yield light Higgs doublets and several fermion mass predictions. The lifting of the degeneracy of these flat directions has not been studied, and will lead to interesting constraints on the form of the supersymmetry breaking terms. The flat directions imply the existence of light supermultiplets which are singlets under the standard model gauge group. Moreover since the continuous $\mathrm{U}(1)$ symmetries are spontaneously broken at $M_{G}$ the theory also includes an invisible axion and possibly several massless Goldstone bosons. These may be problematical in a cosmological context and also require further study.

In this theory we can now calculate corrections to zeroth order fermion mass and mixing angle predictions [4]. Threshold corrections at the GUT

\footnotetext{
" Another possibility, which has been explored recently, is that the pattern of fermion masses arises from a U(1) flavor symmetry which is gauged [14].
} 
scale include corrections to (3) coming from integrating out the heavy $16-\overline{16}$ pairs (only the zeroth order result in a power series in ratios of scales has been included in the analysis of ref. [4]). There are also radiative corrections to gauge and Yukawa parameters; for example, the radiative corrections to the gauge couplings can be significant in the limit $\mathcal{S}_{7} \ll M_{G}$ and thus the low energy measurement of $\alpha, \sin ^{2} \theta_{W}$ and $\alpha_{s}\left(M_{Z}\right)$ could constrain the proton decay rate.

Finally, several groups are now working to obtain SO(10) SUSY GUTs from string theories [15]. We hope that theories like ours can be used as a guide to find a realistic fundamental theory of nature.

\section{Acknowledgements}

We thank R. Barbieri and A. Strumia who participated in an earlier version of this work.

\section{References}

[1] S. Dimopoulos, S. Raby and F. Wilczek, Phys. Rev. D24 1681 (1981); S. Dimopoulos and H. Georgi, Nucl. Phys.B 193150 (1981); L. Ibanez and G.G. Ross, Phys. Lett. 105B 439 (1981); M. B. Einhorn and D. R. T. Jones, Nucl. Phys. B196 475 (1982); W. J. Marciano and G. Senjanovic, Phys. Rev. D 253092 (1982). For recent analyses, see P. Langacker and N. Polonsky, Phys. Rev.D47 4028 (1993); ibid., D49 1454 (1994); M. Carena, S. Pokorski, C.E.M. Wagner, Nucl. Phys.B406 59 (1993).

[2] B. Ananthanarayan, G. Lazarides and Q. Shafi, Phys. Rev.D44 1613 (1991); H. Arason et al., Phys. Rev. Lett. 672933 (1991); S. Kelley, J.Lopez and D. Nanopoulos, Phys. Lett. B274 387 (1992). See also the earlier work of M. Olechowski and S. Pokorski, Phys. Lett.B214 393 (1988). 
[3] A. Brignole, H. Murayama and R. Rattazzi, LBL-35774 (1994). F. Vissani and A. Smirnov, SISSA-63-94-EP (1994).

[4] G. Anderson, S. Dimopoulos, L.J. Hall, S. Raby and G. Starkman, Phys. Rev.D49 3660 (1994).

[5] For early work in this direction, see C.D. Froggatt and H.B. Nielsen, Nucl.Phys.B147 277 (1979); Z.G. Berezhiani, Phys. Lett.129 B 99 (1983), ibid.150 B 177 (1985); S. Dimopoulos, Phys. Lett.129 B 417 (1983); J. Bagger,S. Dimopoulos, H. Georgi and S. Raby, Proceedings of the Fifth Workshop on Grand Unification, Providence, Rhode Island, April 12 - 14, 1984, World Scientific Publishing Co., Singapore (1984).

[6] H. Georgi, Particles and Fields, Proceedings of the APS Div. of Particles and Fields, ed C. Carlson; H. Fritzsch and P. Minkovski, Ann. Phys. 93 193 (1975).

[7] L.J. Hall, in Supersymmetry and Supergravity/Nonperturbative QCD, Proceedings of the Winter School in Theoretical Physics, Mahabaleshwar, India, 1984, edited by P. Roy and V. Singh, Lecture Notes in Physics Vol. 208 (Springer, Berlin, 1984).

[8] S. Dimopoulos and F. Wilczek, Proceedings Erice Summer School, Ed. A. Zichichi (1981). For a recent discussion, see K.S. Babu and S.M. Barr, Delaware preprint BA-94-04 (1994).

[9] Dimopoulos, Carena, Raby and Wagner, private communication.

[10] M. Carena, S. Pokorski, M. Olechowski, and C.E.M. Wagner, "Electroweak Symmetry Breaking and Bottom-Top Yukawa Unification," CERN Theoretical Report No. CERN-Th.7163/94 (LANL hepph/9402253)(1994); R. Rattazzi, U. Sarid and L.J. Hall, "Yukawa Unification: the Good, The Bad and The Ugly," Stanford preprint SU-ITP94-15 (LANL hep-ph/9405313)(1994). 
[11] S. Dimopoulos, S. Raby and F. Wilczek, Phys. Lett.112B 133 (1982); J. Ellis, D.V. Nanopoulos and S. Rudaz, Nucl. Phys.B202 43 (1982). For recent analyses, see R. Arnowitt and P. Nath, Phys. Rev.D49 1479 (1994); J. Hisano, H. Murayama, and T. Yanagida, Nucl. Phys.B402 46 (1993); P. Langacker, "Proton Decay," (LANL hep-ph/9210238) talk given at The Benjamin Franklin Symposium in Celebration of the Discovery of the Neutrino, Philadelphia, PA, 29 Apr - 1 May (1992).

[12] H. P. Nilles and S. Raby, Nucl. Phys. B 198102 (1982).

[13] L.J. Hall, J. Lykken and S. Weinberg, Phys. Rev. D27 2359 (1983).

[14] L. Ibanez and G. G. Ross, Phys. Lett. B 332100 (1994).

[15] S. Chaudhuri, S. Chung and J.D. Lykken, FERMILAB PUB-94/137-T (LANL hep-ph/9405374)(1994); G. Cleaver, Talk given at Paschos 94. 


\section{Figure Captions}

1. Diagrams which generate the operators of equation 1.

2. A diagram which generates a one loop contribution to $\mu$ by integrating out superheavy fields. 